\title{
Detecting phase synchronization in chaotic non-coherent Rössler systems using Discrete Complex Wavelet Approach (DCWA): preliminaries results
}

\author{
Maria Teodora Ferreira ${ }^{1}$ \\ Margarete O. Domingues ${ }^{2}$ \\ Elbert E. N. Macau ${ }^{3}$ \\ Associate Laboratory for Computing and Applied Mathematics - LAC, \\ Brazilian National Institute for Space Research - INPE, Brazil
}

\begin{abstract}
Phase synchronization in non-identical coupled chaotic systems appears for some conditions in which weak coupling causes the systems time evolution to lock in phase to one another, while their amplitudes remain chaotic and uncorrelated. To identify this phenomenon, given a signal it is necessary to measure properly its phase. In this work, we applying our Discrete Complex Wavelet Approach (DCWA) for phase assignment, with aim to analyse two coupled chaotic Rössler systems in non-phase coherent regime. Our DCWA is based on the Dual-Tree Complex Wavelet Transform (DT-CWT), which is a form of a discrete wavelet transform that generates complex coefficient by using a dual-tree wavelet filters to obtain their real and imaginary parts. Due to its multi-scale properties in the context of phase characterization, it is possible to obtain very good results from scalar time series, even with chaotic systems.
\end{abstract}

Keywords. Chaos, Phase Synchronization, Synchronization in chaotic systems, Wavelet, Rössler systems.

\section{Introduction}

The phenomenon of synchronization has been observed in various natural systems, such as in heart cells, applause, flashing of the South-East Asian fireflies, chirping of crickets, and more $[2,6]$. Initially, the synchronization was studied in periodic systems. For theses systems, basically, synchronization is understood as the mutual adjustment of the oscillations of periodic oscillators due to some sort of interaction between them [13]. The generalization of the concept of synchronization, allowing to encompass chaotic system, was presented in $[1,11,13,14,17]$.

\footnotetext{
${ }^{1}$ mteodoraf25@gmail.com

${ }^{2}$ margarete.domingues@inpe.br

${ }^{3}$ elbert.macau@inpe.br
} 
In this work our interest is phase synchronization, which occurs mainly for weak coupling with the amplitudes of the oscillators remaining uncorrelated while their phases evolve in step with each other over time [17]. Detecting phase synchronization of chaotic systems requires a clear and unambiguous phase assignment for the oscillators in order to test the condition ( [13])

$$
\Delta \phi(t)=\left|\phi_{1}(t)-\phi_{2}(t)\right| \leq \text { const }<2 \pi,
$$

wherein $\phi_{1}(t)$ and $\phi_{2}(t)$ are the phases of systems 1 and 2, respectively.

Several methods have been proposed for phase assignment, for example, Hilbert transform [15], Poincaré surface of section [13], curvature and recurrence plots [10,16], localized sets [12], a method based on least squares analysis [5], short-time Fourier transforms and the continuous complex wavelet transform methodologies [7,9]. However, none of these methods works properly for all the situations. The majority of them have as the input the projection of the attractor on a proper surface of section; present good results only in phase-coherent regime and generally are not directly applicable to experimental time series. In particular, the methodologies that use continuous complex wavelet transform are based on the complex Morlet wavelet to obtain the phase of a time series yielding good results for systems in phase-coherent regime [7,9]. However, these approach implies a high computational cost and the results may be not easy to understand, due to its redundancy framework when applied to large time series and in chaotic systems.

To overcome these difficulties, in [4] we propose our DCWA based on the Dual-Tree Complex Wavelet Transform (DT-CWT) for phase assignment. The DT-CWT is a transform that employs two real Discrete Wavelet Transforms (DWT). The main qualities of this transform are that it is nearly shift invariant, limited redundancy and reduced computational cost $[3,8,19]$. In this work, our DCWA is employed in the analysis of two chaotic Rössler systems under non-phase-coherent regime.

The remainder of this paper is organizes as follows. In Section 2 we present our DCWA. Subsequently, in the Section 3, the analysis of the results are commented. In the last Section 4, we provide the conclusion and future works.

\section{Methodology}

In this section, we describe how to calculate the phase using our DCWA. Further details can be seen in [4].

In order to calculate the phase of a system using the Discrete Complex Wavelet Approach (DCWA), the time series of a scalar variable $x$ of the system is analysed by the DT-CWT. For more details of DT-CWT see $[8,19]$. The Figure 1(a) shows a scheme of this analysis. As the output this transformation, we have the time series of the complex wavelet coefficients $\boldsymbol{d}^{j}$ at each scale $j$. With these coefficients, the energy $E^{j}$ at each scale $j$ is calculated as the square of the modulus of complex wavelet coefficients, i.e, $E^{j}(n)=\left|\boldsymbol{d}^{j}(n)\right|^{2}$. After that, the global wavelet spectrum is computed as follows:

$$
\mathbb{E}^{j}=\sum_{n} E^{j}(n)
$$


In the next step, we take the scale $J$ in which the global wavelet spectrum energy is the maximal, i.e., $\mathbb{E}^{J}=\max _{j} \mathbb{E}^{j}$. Therefore, this scale $J$ is used to extract the phase time series, according to the equation

$$
\phi^{J}(t)=\operatorname{atan} 2\left(\underline{d}^{J}, \bar{d}^{J}\right) .
$$

The atan2 is the arctangent function with two arguments; $\underline{d}^{j}$ is the imaginary part of the complex wavelet coefficient in the scale $J$ and $\bar{d}^{J}$ is the real part of the complex wavelet coefficient in the scale $J$.

Consider now two systems with time series $x_{1}$ and $x_{2}$. The method described above applied to both of them can be viewed in Figure 1(b). Whenever $J_{1}$ was different from $J_{2}$ we chose the scale $J=\min \left(J_{1}, J_{2}\right)$. This choice was based on the fact that we chose the larger phase time series. Subsequently, the phase time series of each system, $\phi_{1}^{J}$ and $\phi_{2}^{J}$ is calculated. The instantaneous phase difference between the systems is computed as $\Delta \phi^{J}=\left|\phi_{1}^{J}-\phi_{2}^{J}\right|$, which allows one to check for the phase synchronization condition $\Delta \phi^{J}=\left|\phi_{1}^{J}-\phi_{2}^{J}\right|<$ constant.

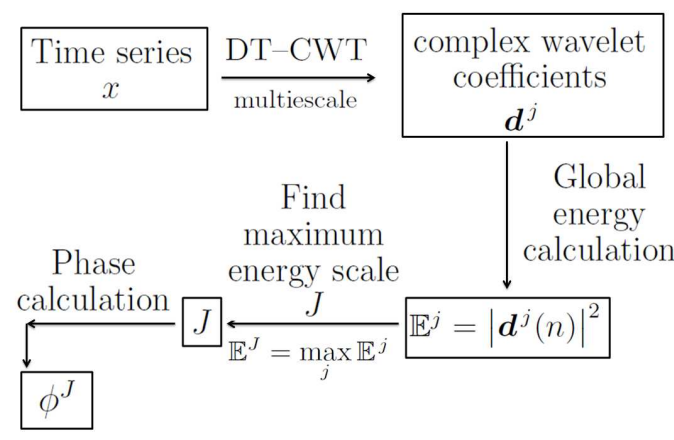

(a)

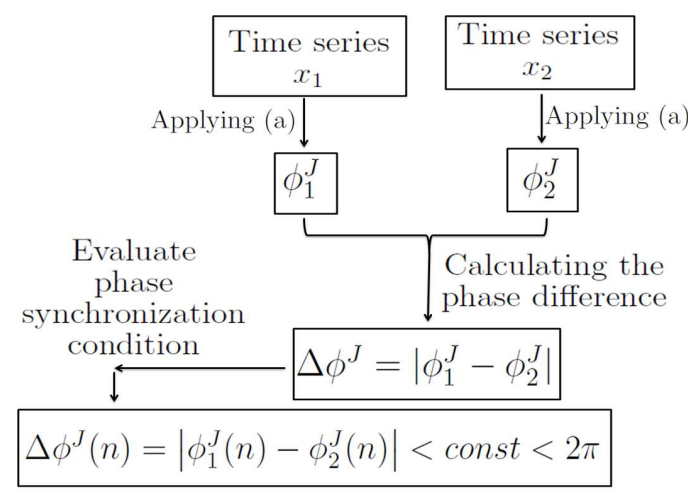

(b)

Figure 1: Schematic representation of our DCWA. In Subfig. (a), a single times series $x$ is considered and (b) the application of our DCWA in two times series, $x_{1}$ and $x_{2}$.

\section{Application and Results}

Here, our methodology is applied for time series generated by two non-identical chaotic Rössler systems [18] in non-phase-coherent regime and coupled bidirectionally by variable $y$. We use the Runge-Kutta 4th order method with integration time step equal to 0.01 and the number of points in the time series is $N=2^{23}$. The system is given by equations (4) and the parameters considered are based in $[5,16]$.

$$
\begin{aligned}
& \dot{x}_{1,2}=-\omega_{1,2} y_{1,2}-z_{1,2} \\
& \dot{y}_{1,2}=\omega_{1,2} x_{1,2}+0.2925 y_{1,2}+\eta\left(y_{2,1}-y_{1,2}\right) \\
& \dot{z}_{1,2}=0.4+z_{1,2}\left(x_{1,2}-8.5\right) .
\end{aligned}
$$


wherein $\omega_{1}=0.98$ and $\omega_{2}=1.02$ defines the mismatch in the natural frequencies and parameter $\eta$ is the intensity of coupling between these two systems.

In this application, three different intensities of coupling are considered: weak in the value of $\eta=0,05$, medium with $\eta=0,17$, and strong with $\eta=0,23$. These coupling strengths were empirically adjusted to obtain three possible states for systems analysis: not phase synchronized, phase-slips and in phase synchronization.

The Figure 2 illustrates in (a, c, e) the global wavelet spectrum for each coupling intensities: $\eta=0.05, \eta=0.17$ and $\eta=0.23$, respectively.

Note that for the three coupling intensities, the maximum energy scale is the $J=9$. Therefore, the phases are calculated in this scale, denoted by $\phi_{x_{1}}^{J=9}$ and $\phi_{x_{2}}^{J=9}$. The phases are shown in Fig.2 (b, d, f). Despite the presence of some small perturbations, the phase is increasing over time, which is consistent with the phase definition.

The phase difference between the two systems are presented in Fig. $2(\mathrm{~g}, \mathrm{~h})$; wherein are used in (g) our DCWA $\left(\Delta \phi_{x}^{J=9}\right)$ and $(\mathrm{h})$ the arctangent method, $\left(\Delta \phi^{\text {arco-tangente }}\right)$ $([10,16])$ by way of comparison. For small intensity of coupling $\eta=0.05$, the phase difference increases with time, which characterizes the absence of phase synchronization. Note in this case that both our DCWA and arctangent method correctly detects that the systems are not synchronized in phase, as can be seen in Fig. $2(\mathrm{~g}, \mathrm{~h})$. If the coupling strength is increased to $\eta=0.17$, our DCWA detects seven regions in which phase-slips appear. Note that arctangent method $(2(\mathrm{~h}))$ detects only three regions of phase-slips. When $\eta=0.23$, phase synchronization arises and both methods are able to detect correctly this phenomenon. 


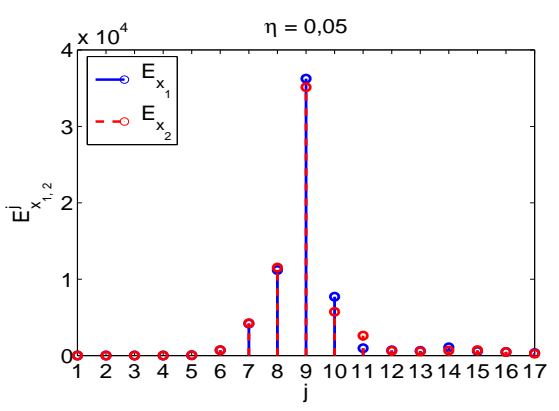

(a)

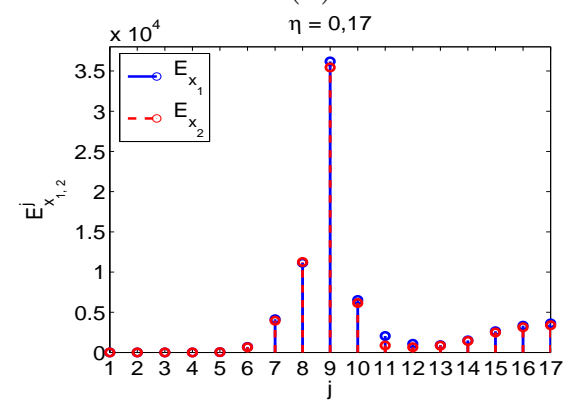

(c)

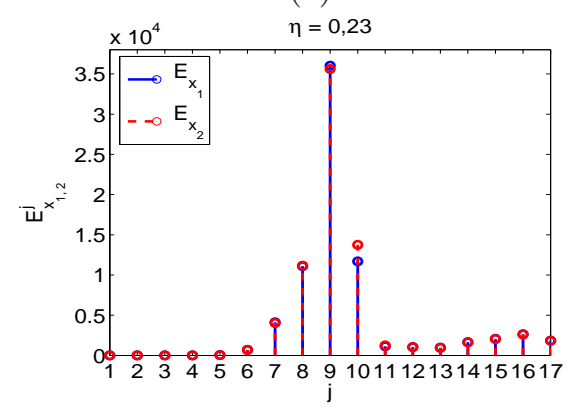

(e)

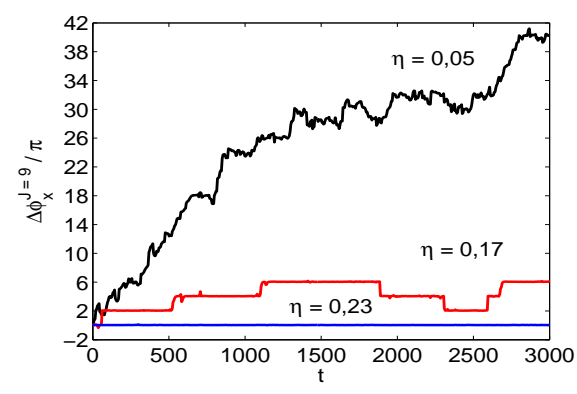

(g)

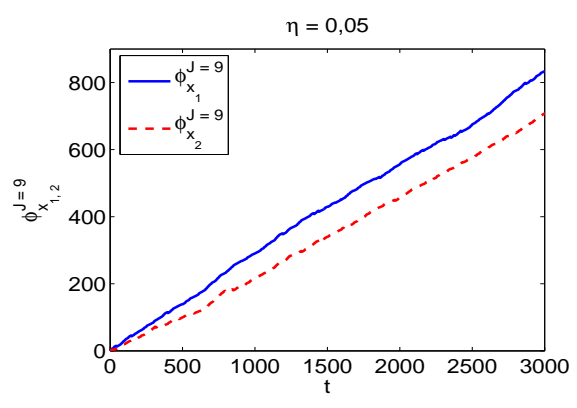

(b)

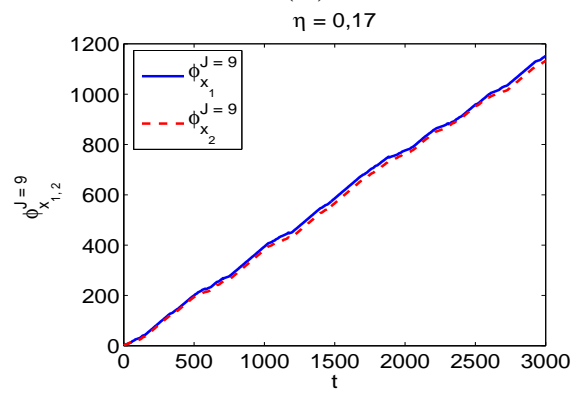

(d)

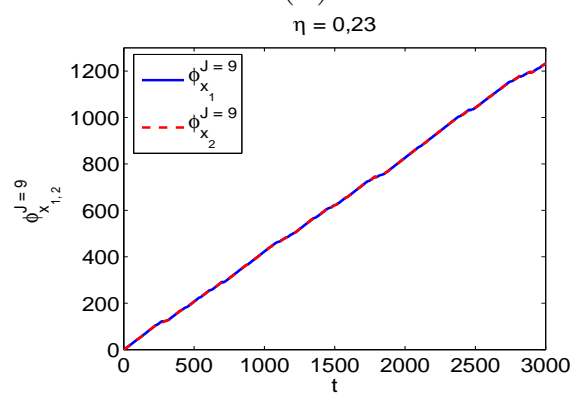

(f)

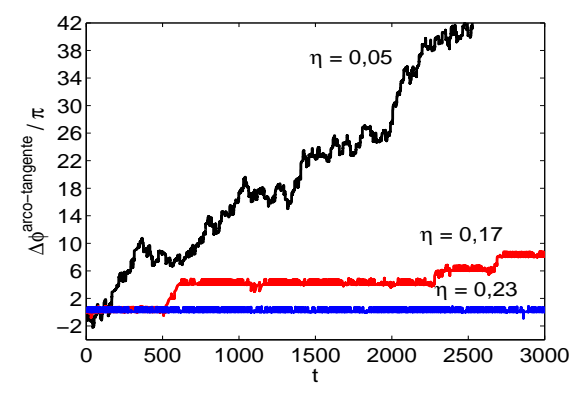

(h)

Figure 2: In (a, c, e) the global wavelet spectrum for each coupling intensities: $\eta=0.05, \eta=0.17$ and $\eta=0.23$, respectively. The phases for each coupling are shown in (b, $\mathrm{d}, \mathrm{f})$, respectively. The phase difference between the two systems are shown in (g) using our DCWA and (h) the arctangent method. 


\section{Conclusion}

In this paper we applied our DCWA in the analysis of two chaotic Rössler systems under non-phase-coherent regime. The accuracy of our DCWA is demonstrated by the results obtained to detect phase synchronization between two coupled chaotic Rössler systems in non-phase-coherent regime. The our method was able to correctly analyse the not phase synchronized, phase-slips and phase synchronization. Moreover, our DCWA requires only a time series of the system without the need of reconstruction of the attractor, a very convenient feature, especially in the case of experimental data.

\section{Acknowledgements}

This work was supported by CAPES, CNPq (grants 309667/2013-5, 306828/2010-3, 483226/2011-4, 10571/13-2, 304582/2010-7) and FAPESP (grant 2011/50151-0).

Nick Kingsbury by available the DT-CWT algorithm and presented two mini-courses on this subject in the WWlet/CNMAC 2012.

\section{References}

[1] S. Boccaletti, J. Kurths, G. Osipov, D. L. Valladares and C. S. Zhous, The synchronization of chaotic systems, Physics Reports, vol. 366, n. 1-2, 1-101, (2002).

[2] J. Buck, Synchronous rhythmic flashing of fireflies II, The Quarterly Review of Biology, vol. 63, n. 3, 265-289, (1988).

[3] I. Daubechies, Wavelet transform, time-frequency localization and signal analysis, IEEE Transactions on Information Theory, vol. 36, n. 5, 961-1005, (1990).

[4] M. T. Ferreira, C. B. N. Freitas, M. O. Domingues and E. E. N. Macau, The discrete complex wavelet approach to phase assignment and a new test bed for related methods, Chaos: An Interdisciplinary Journal of Nonlinear Science, vol. 25, 013117, (2015).

[5] R. Follmann, E. E. N. Macau and E. Rosa, Phase detection of chaos, Physical Review E, vol. 83, 0162091-0162096, (2011).

[6] L. Glass, Synchronization and rhythmic processes in physiology, Nature, vol. 410, n. $6825,277-284,(2001)$.

[7] A. E. Hramov, A. A. Koronovskii and Yu. I. Levin, Synchronization of chaotic oscillator time scales, Journal of Experimental and Theoretical Physics, vol. 100, n. 4, 784-794, (2005).

[8] N. G. Kingsbury, The dual-tree complex wavelet transform: a new efficient tool for image restoration and enhancement in 9th European Signal Processing Conference (EUSIPCO 1998), 319-322, (1998). 
[9] A. A. Koronovskii, M. K. Kurovskaya and A. E. Hramov, Relationship between phase synchronization of chaotic oscillators and time scale synchronization, Technical Physics Letters, vol. 31, n. 10, 847-850, (2005).

[10] J. Kurths, M. C. Romano, M. Thiel, G. V. Osipov, M. K. Ivanchenko, I. Z. Kiss and J. L. Hudson, Synchronization analysis of coupled noncoherent oscillators, Nonlinear Dynamics, vol. 44, n. 1, pp. 135-149, (2006).

[11] L. M. Pecora and T. L. Carrol, Synchronization in chaotic systems, Physical Review Letters, vol. 64, n. 8, 821-824, (1990).

[12] T. Pereira, M. S. Baptista and J. Kurths, General framework for phase synchronization through localized sets, Physical Review E, vol. 75, n. 2, 026216, (2007).

[13] A. S. Pikovsky and M. G. Rosenblum, Phase synchronization of regular and chaotic self-sustained oscillators, Synchronization: Theory and Application, NATO Science Series, vol. 109, 187-219, (2003).

[14] A. Pikovsky, M. Rosenblum and J. Kurths, Synchronization: a universal concept in non-linear sciences, New York: Cambridge University Press, (2003).

[15] M. L. V. Quyen, J. Foucher, J. Lachaux, E. Rodriguez, A. Lutz, J. Martinerie and F. J. Varela, Comparison of Hilbert transform and wavelet methods for the analysis of neuronal synchrony, Journal of Neuroscience Methods, Elsevier, vol. 111, n. 2, 83-98, (2001).

[16] M. C. Romano, M. Thiel, J. Kurths, I. Z. Kiss and J. L. Hudson, Detection of synchronization for non-phase-coherent and non-stationary data, Europhysics Letters, vol. 71 , n. 3, 466-472, (2005).

[17] M. Rosenblum, A. Pikovsky, J. Kurths, C. Schäfer and P. A. Tass, Chapter 9 Phase synchronization: From theory to data analysis in Handbook of Biological Physics, vol. 4, 279-321, (2001).

[18] O. E. Rössler, An equation for continuous chaos, Physics Letters A, vol. 57, n. 5, 397-398, (1976).

[19] I. W. Selesnick, R. G. Baraniuk and N. G. Kingsbury, The dual-tree complex wavelet transform, IEEE Signal Processing Magazine, vol. 22, n. 6, 123-151, (2005). 